\title{
Experience with Ceftriaxone in the Treatment of Pulmonary Infections
}

\author{
Faroque A. Khan, M.D., FCCP, FACP \\ Joseph J. Guarneri, PhD \\ Rajinder Chitkara, M.D., FCCP, FACP \\ Phyllis DellaLatta, PhD \\ Vincent LaBombardi, PhD \\ Parvez Mir, M.D. \\ Suhail Raoof, M.D.
}

DOI: http://dx.doi.org/10.5915/17-1-12717

\section{Abstract:}

This paper describes the experience with a new third generation cephalosporin, Ceftriaxone, in the treatment of 60 patients with bacterial pulmonary infections. The unique pharmacokinetics of Ceftriaxone allow its usage twice a day in contrast to the 4 to 6 times a day for comparable antibiotics, with potential cost savings. Administered twice a day the overall cure rate was $94 \%$ with no serious side effects. A brief review of the clinical application of Cephalosporins in the treatment of infections is included.

\section{Introduction}

Beta-Lactam antibiotics have had triumphs during the past 30 years. Illnesses such as bacterial endocarditis and fulminant meningitis, which had a mortality of $100 \%$, respond to penicillin $\mathrm{G}$, with not only a decrease in mortality but also a major decline in morbidity. Many new penicillins and cephalosporins have been developed, and many compounds are still being clinically evaluated. The precise clinical use of new beta-lactam agents is not easy to define, particularly the use of cephalosporins as the primary treatment of serious infection. In spite of the confusion about the use of these agents, cephalosporins have been the most widely used antibiotics to treat hospitalized patients ${ }^{1},{ }^{2}$.

Many factors have contributed to the proliferation of new beta-lactam compounds. There has been increased resistance of gram-negative bacteria to existing compounds and an increased awareness of the toxic potential of other antimicrobial agents, particularly the aminoglycosides. Finally, advances in chemistry have made possible the production of new cephalosporin or oxacephem compounds with extraordinary in-vitro activity ${ }^{3}$.

Cephalosporins consist of a dihydrothiazolidine ring fused with a four member beta-lactam ring. It is possible to make major modifications of the basic nucleus, and as a result of these modifications there has been a proliferation of the new cephalosporins. The cephalosporins can be classified into three " generations" based on their activity against gram-negative organisms. All "first-generation" cephalosporins have a similar spectrum, including many gram-positive cocci (but not enterococci or methicillin-resistant Staphylococcus aureus), Escherichia coli, Klebsiella pneumoniae, and Proteus mirabilis.

The "second-generation" cephalosporin have broader in vitro activity against gram-negative bacteria. Cefamandole and cefaclor have increased activity against Haemophilus influenza and some gram-negative bacilli. Cefoxitin has improved activity against Bacteroides fragilis, Neisseria gonorrhoea, and some aerobic gram-negative bacilli.

The "third-generation" cephalosporin are less active than older cephalosporins against gram positive cocci, but more active against enteric gram-negative bacilli, including nosocomically acquired stains resistant to multiple antibiotics, and moderately active against $\mathbf{P}$. aeruginosa. These agents are highly active against $\mathbf{H}$. influenzae and N. gonorrhoea, including penicillinaseproducing strains. They are moderately active against anaerobes, but often less so than metronidazole (Flagyl; others), chloramphenicol (Chloromycetin; others), clindamycin (cleocin), or cefoxitin. Cefotaxime is more active in vitro than moxalactam or cefoperazone against anaerobes. Cefoperazone is less active than the other two against many gram-negative bacilli, but more active against Pseudomonas ${ }^{2}$. All the currently available cephalosporins, with their comparative data and costs are listed in Table 1. This paper reports our experience with a new third line cephalosporin, Ceftriaxone (RO 13-9904), in the treatment of pulmonary infections at Queens Hospital Center (QHC) in New York.

\section{Materials and Methods:}

This clinical study was designed to determine the efficacy of a new third generation cephalosporin, Ceftriaxone (RO 13-9904) 2gms IV BID, for the treatment of serious pulmonary infections. As a control, a currently available second generation cephalosporin, Cefoxitin sodium, 2gms IV Q6h was used. The patients were assigned to the drugs according to a randomization number determined by a predetermined code. Sixty patients from the pulmonary service at Queens Hospital Center were enrolled for the study from November 1981

From the Division of Pulmonary Medicine, Department of Medicine and Division of Microbiology, Department of Laboratories, Queens Hospital Center Affiliation of the Long Island Jewish-Hillside Medical Center and School of Medicine, Health Sciences Center, State University of New York at Stony Brook

Presented at the 17th Annual Convention of the Islamic Medical Association in St. Louis, Missouri, August 10-12, 1984

Address all correspondence to: Dr. Faroque Khan Division of Pulmonary Medicine, Queens Hospital Center 82-68 164th Street, Jamaica, New York 11432 


\section{CEPHALOSPORINS}

Table 1:

Drug

Comparative Data

Most experience, painful

on IM administration

Less painful on IM injection

(Ancef-SKF)

(Kefzol-Lilly)

Cephapirin sodium

(Cefadyl-Bristol)

Cephradine

(Velosef-Squibb)

Cephalexin

(Keflex-Dista)

Cephradine

(Anspor-SKF)

(Velosef-Squibb)

Cefadroxil

(Duricef-Mead Johnson)

(Ultracef-Bristol)

\section{SECOND GENERATION}

Cefamandole

(Mandol-Lilly)

Cefoxitin

(Mefoxin-MSD)

Cefaclor

(Ceclor-Lilly)

THIRD GENERATION

Cefotaxime sodium

(Claforan-Hoechst-Roussel)

Moxalatam disodium

(Moxam-Lilly)

Cefoperazone

(Cefobid-Roerig)

Ceftizoxime sodium

(Cefizox-SFK)

Cefonicid sodium

(Monocid-SKF)
More painful than cefazolin

on injection

Available for oral and

parenteral use

Most experience for oral therapy

Equivalent to cephalexin

No advantage in spectrum; prolonged excretion permits

bid dose; more limited indications

More active against $\mathrm{H}$.

influenzae and some gram negative bacilli; not recommended for meningitis

More active against $\mathbf{B}$.

fragilis, some gram negative bacilli, and $\mathbf{N}$. gonorrhea

Greater activity against

H. influenzae; may be useful in otitis media

Increased activity against

gram-negative bacilli; good CSF penetration

Increased activity against gram-negative bacilli; least active 3 rd generation vs gram-positive; good CSF penetration

Increased activity against

Pseudomas aeruginosa; no accumulation in renal failure

Increased activity against gram negative bacilli

Increased acivity against gram negative bacilli
Daily

Cost of

Adult

10 Days

Route Dosage

Treatment
IM, IV 2-12gm/day $\$ 429.14$ q4-6h

IM, IV 1-6gm/day q6-8h
IM, IV 2-12 $\mathrm{gm} /$ day q4-6h

IM, IV

$2-8 \mathrm{gm} / \mathrm{day}$

400.20 q4-6h

Oral

$1-4 \mathrm{gm} /$ day

78.67 q6h

Oral 1-4gm/day q6h

69.52

77.22

Oral

$1-2 \mathrm{gm} /$ day q12-24h

40.35

44.28

IM, IV

$1.5-12 \mathrm{gm} / \mathrm{dy}$ q4-8h

785.76

IM, IV

3-12gm/day

981.74 q4-8h

Oral

$0.75-4 \mathrm{gm} / \mathrm{dy}$ q8h

120.59

IM, IV

$2-12 \mathrm{gm} /$ day

1199.40 q4-6h

IM, IV 2-12gm/day

1464.54

IM, IV

$2-12 \mathrm{gm} /$ day

1353.60 q6-8h

IM, IV

4-12gm/day

1200.00 q6-8h

IM, IV

$1-2 \mathrm{gm} /$ day $\mathrm{q} 24 \mathrm{~h}$

- Cost to pharmacist as listed in Drug Topics Red Book 1983 and May 1983 update. 
to December 1982. All enrolled patients gave informed consent, had routine hospital investigations including a complete blood count with differential, chest $\mathrm{x}$-rays, urine analysis and $\mathrm{SMA}_{18}$, in addition to sputum and blood cultures. To be eligible for evaluation in the protocol, patients had to receive a minimum of five days of antibiotics. In cases where a pathogen was isolated from a specimen, antimicrobial susceptibility tests were performed by the standardized disc diffusion method ${ }^{4}$ using 30ug Ceftriaxone and Cefoxitin discs. The zone size interpretive criteria for these drugs were as follows: a) Ceftriaxone $-16 \mathrm{~mm}$, sensitive; $13-15 \mathrm{~mm}$, intermediate and $-12 \mathrm{~mm}$, resistant and b) Cefoxitin $-18 \mathrm{~mm}$, sensitive; $15-17 \mathrm{~mm}$, intermediate and $-14 \mathrm{~mm}$, resistant. For final evaluation of the clinical results the following criteria were considered: a) Clinical response - fever, cough, sputum, b) Laboratory - white blood cell count WBC, c) Radiologic - serial x-ray, d) Microbiologic response. The clinical outcome was classified as a) Clinical cure - clinical findings subsiding in a reasonable period of time with no clinical evidence of infection at the time the drug was discontinued, nor during follow up; b) Clinical improvement - clinical findings subsiding significantly in a reasonable period of time but with incomplete resolution of clinical evidence of infection, or relapse during follow up, possibly related to underlying disease stage; c) Failure - no apparent response to therapy; d) Impossible to evaluate.

\section{Results of Study:}

Of the 60 patients enrolled, 6 were withdrawn due to lack of completion of the minimum 5 days therapy. The remaining 54 patients form the basis of this report. Of these 54 patients, 20 had no bacteriologic confirmation although all of them had the clinical features of bacterial pulmonary infection. Of these 20,11 were in the Ceftriaxone arm and 9 in the Cefoxitin sodium arm of the study. In the Ceftriaxone group, of the 11 patients 10 had a clinical cure while in the Cefoxitin sodium group, of the 9 patients 6 were considered cured and the remaining 3 had no response.

Of the 34 patients with bacteriologic confirmation, 17 were in the Ceftriaxone arm and 17 in the Cefoxitin arm. The microbiologic data of these 34 patients along with their sensitivity patterns are shown in Table 2. There were a total of 41 sputum and 17 blood isolates recovered from these 34 patients. The isolates included Acinetobactercalcoaceticus var anitratum (5), Haemophilus influenzae (10), Proteus mirabilis (3), Enterobacter cloacae (3), Escherichia coli (1), Klebsiella pneumoniae (1), Pseudomonas aeruginosa (3), Streptococcus pneumoniae (27), Beta hemolytic Streptococci Group A (1) S. aureus (1), S. epidermis (3). As seen in Table 2 , the sensitivity pattern was similar in all gram positive cocci. The only significant difference was in the sensitivity pattern of the gram negative bacteria. Specifically 3 of 4 Acinetobactercalcoaceticus var antitratum isolates and 2 of 3 Pseudomonas aeruginosa isolates were sensitive to Ceftriaxone but resistant to Cefoxitin. In both groups of patients the results of the antibiotic regimen were comparable. In the Ceftriaxone group 1 out of 17 patients studied was considered a failure while the remaining 16 were considered cured. In the 17 Cefoxitin treated patients, 15 were considered cured while 2 were treatment failures.

\section{Discussion}

Cephalothin (Keflin) was the first cephalosporin introduced for clinical use in 1964. Since then several modifications of the basic structure of the cephalosporin nucleus have been made leading to a rapid proliferation of the available cephalosporins (Table 1). Each succeeding generation is claimed to have broader antibacterial coverage, however the costs of administer-

Table 2:

Types of Bacteria Isolated From Study Patients ${ }^{\mathrm{a}}$ With Pneumonia

\begin{tabular}{|c|c|c|c|}
\hline Bacteria & Site & Ceftriaxone & Cefoxitin \\
\hline Streptococcus pneumoniae & sputum (15) & $\begin{array}{c}\text { \% Sensitive } \\
100\end{array}$ & $\begin{array}{l}\text { \% Sensitive } \\
100\end{array}$ \\
\hline Streptococcus pneumoniae & blood (12) & 100 & 100 \\
\hline Beta Streptococci Group A & sputum (1) & 100 & 100 \\
\hline Staphylococcus aureus & sputum (1) & 100 & 100 \\
\hline Staphylococcus epidermis & blood (3) & 100 & 100 \\
\hline Haemophilus influenzae & sputum (9) & 100 & 100 \\
\hline Haemophilus influenza & blood (1) & 100 & 100 \\
\hline Acinetobacter calcoaceticus & & & \\
\hline var anitratum & sputum (4) & 75 & 0 \\
\hline Acinetobacter calcoaceticus & & & \\
\hline var anitratum & blood (1) & 100 & 0 \\
\hline Proteus mirabilis & sputum (3) & 100 & 67 \\
\hline Pseudomas aeruginosa & sputum (3) & 67 & 33 \\
\hline Escherichia coli & sputum (1) & 100 & 100 \\
\hline Klebsiella pneumoniae & sputum (1) & 100 & 100 \\
\hline Enterobacter cloacae & sputum (3) & 100 & 100 \\
\hline
\end{tabular}

a Multiple isolates with identical antibiograms from the same patient are not included. 
ing these drugs can be prohibitive as shown in Table 1. One possible way of reducing the cost of antibiotic therapy is to reduce the frequency of administration. With this in mind newer antibiotics are being marketed with a daily recommended usage of once or twice a day. In view of its unique properties, Ceftriaxone belongs to this group of antibiotics ${ }^{3}{ }^{4}$. Ceftriaxone is a highly polar acidic molecule that binds with protein to a much greater degree than the other agents of this type. Protein binding ranges from $83 \%$ at $30 \mathrm{ug} / \mathrm{mL}$ to $96 \%$ at concentrations of less than $1 \mathrm{ug} / \mathrm{mL}$. Thus the pharmacokinetics are nonlinear. Serum levels of $150 \mathrm{ug} / \mathrm{mL}$ follow infusion of $1 \mathrm{~g}$ over 30 minutes with levels of $35 \mathrm{ug} / \mathrm{mL}$ at 12 hours and $15 \mathrm{ug} / \mathrm{mL}$ at 24 hours. Administration of $0.5 \mathrm{~g}$ intramuscularly yields serum levels of $50 \mathrm{ug} / \mathrm{mL}$ at 6 hours and $8 \mathrm{ug} / \mathrm{mL}$ at 24 hours. The serum half-life is about 8 hours. There is no renal tubular secretion of Ceftriaxone as shown by the failure of probenecid to alter plasma levels or half-life. Urinary recovery is only $60 \%$, and because there is no major metabolism of Ceftriaxone, $35 \%$ to $40 \%$ of the antimicrobial agents is eliminated by biliary secretion. The drug does not accumulate in patients with renal failure unless there is combined hepatic dysfunction. Ceftriaxone shows excellent tissue distribution and enters the cerebrospinal fluid in the presence of inflammation, producing levels of $1 \mathrm{ug} / \mathrm{mL}$ to $15 \mathrm{ug} / \mathrm{mL}^{3,5}$.

While the treatment of pulmonary infections with known pathogens is simple, in clinical practice the bacterial etiology of an infection is not established in a large number of patients. In our study, of the $60 \mathrm{pa}-$ tients who fulfilled the clinical criteria of bacterial pulmonary infections, 20 did not have any bacterial pathogens isolated and 16 of them responded to the antibiotics given. Our experience with lack of isolation of bacteria in 20 of 60 patients is similar to the report from John Hopkins University where $47 \%$ of the sputum culture in patients suspected of having pneumonia were reported as either "normal flora" or "poor specimen"'6. Barrett-Connor has asserted that a sputum culture is not valuable for the diagnosis of pneumococcal pneumonia? When sputum is expertly processed the yield of pneumococcus increases ${ }^{8,9}$. The reasons for the variability of both the Gram stain and sputum culture seems to be related to a) inadequacy of the sputum specimen, b) technical problems, c) inability of a single bacteriologic test to detect all causative organisms, and d) the administration of antibiotics before obtaining the culture. This often leads to difficulty in ascertaining the cause of pneumonia particularly of the community acquired type $\mathrm{e}^{10}$. In nosocomical pulmonary infections usually several bacteria are isolated from the sputum and differentiating colonizers from the true pathogens becomes a real clinical and therapeutic challenge. Thus in the practice of medicine, antibiotics are often prescribed without an established bacteriologic etiology.

Although cephalosporins are not in the first column of any manual of chemotherapy, their broad antibacterial action had led to their widespread use. In most hospitals about $5 \%$ of the total budget is used for drug costs with parenteral antibiotics representing $30-50 \%$ of all antibiotic costs. One possible method of reducing the costs of these antibiotics is by administering them at reduced frequency. In our study Ceftriaxone worked quite well when used twice daily with a cure rate of $94 \%$. In a study reported from Lausanne, Switzerland ", Ceftriaxone was administered once daily in 127 patients with 132 episodes of infections. Eighty infections had previously been treated unsuccessfully with other antimicrobials to which the pathogens were most often resistant. Sixty-five episodes were initially treated with two daily injections until there was an improvement in the patient's clinical condition and then received a single daily dose. The remainder of the 67 infections were treated from the start by a single daily injection. The results in the two groups were similar. One hundred and fifteen infections $(86 \%)$ were cured or improved, 10 $(8 \%)$ did not respond to therapy or recurred, and eight $(6 \%)$ were not evaluable. The treatment was well tolerated, even by the 18 patients who received the drug for more than 4 weeks. The administration of a single daily dose instead of 4 doses as with standard antibiotic regimens produced a saving of Sfr $84,000(\$ 42,000)$ in the 127 patients. The single daily dose also made it possible to treat 25 of the 127 severely ill patients as outpatients, with a saving of Sfr $388,500(\$ 195,000)$ with respect to the hospital costs that would have been incurred for the same time period.

Cefonicid sodium (Monocid SKF) has also been recently marketed in USA for once a day use, which reduces the significant costs associated with the delivery of multiple daily doses of parenteral cephalosporins. These delivery costs can and often do exceed drug acquisition costs. In depth studies have quantitated these savings which can add up to significant amounts of money ${ }^{12}$.

In conclusion, our study demonstrates that Ceftriaxone used twice a day was curative in $94 \%$ of patients with an established etiology of pneumonia caused by either Gram positive or Gram negative bacteria and was also curative in 10 of 11 patients with pneumonia of undetermined etiology. Others ${ }^{11}$ have shown that once a day regimen with Ceftriaxone is also effective.

The enlarged antibacterial spectrum of these new cephalosporins, combined with excellent pharmacokinetic properties and retention of the safety of the older agents of this class, suggests that these new drugs could be used in many cases of pulmonary infections. Low inhibitory levels and pharmacological properties permit twice daily dosing with Ceftriaxone, Moxalactam, Cefoperazone and once daily therapy with Cefonicid and Ceftriaxone. But the manner in which the new agents should replace older agents such as Cefazolin or the aminoglycosides is not completely established. Which agent or agents is the best for a hospital to put in its formulary is not known and may vary with the specific patient population under consideration. These questions remain unanswered because the drugs have not been directly compared. Cost factors may make use of the better and newer agents an attractive prospect par- 
ticularly in view of the health care system. Inappropriate use of these agents can cause selection of bacteria which are resistant to them. Some uses of these drugs would include nosocomical urinary tract infections due to multiresistant bacteria, osteomyelitis due to gramnegative bacilli resistant to older agents, and the rare patient with meningitis due to E. coli, Klebsiella or Proteus. These agents are not to be considered the first line of therapy for community-acquired pneumonia, endocarditis, or outpatient urinary tract infections. None of these agents adequately inhibits $\mathbf{S}$. faecalis or $\mathbf{S}$. faecium, the true enterococci, or methicillin-resistant staphylococci.

\section{REFERENCES:}

1. Neu HC: The new beta-lactamase stable cephalosporins. Ann Int Med 97:408-19, 1982.

2. The Medical Letter. Published by the Medical Letter Inc, New Rochelle, N.Y: Choice of cephalosporins - 25 (issue 637), 58-60, 1983.

3. Stoeckel K, McNamara PJ, Brandt R, PlozzaNottebrock H, Ziegler WH: Effect of concentration dependent plasma protein binding of ceftriaxone kinetics. Clin Pharmacol Ther 29:650-57, 1981.

4. Barry AL, Thornsberry C: Susceptibility testing: diffusion test procedures. pp 463-74. In E.A. Lennette, A. Ballows, W.J. Hausler, Jr., and J.P. Truant (ed.). Manual of clinical microbiology, 3rd ed. American Society for Microbiology, Washington, D.C., 1980.

5. Patel IH, Miller K, Weinfeld R, Spicehandler J. Multiple intravenous dose pharmacokinetics of ceftriaxone in man. Chemotherapy 27:47-56, 1981.

6. Dans PE, Charache P, Fahey M, Otter SE: Management of pneumonia in the prospective payment era: A need for more clinician and support service interaction. Arch Int Med 144:1392-97, 1984.
7. Barrett-Connor E: The non value of sputum culture in the diagnosis of pneumococcal pneumonia. Am Rev Resp Dis 103:845-48, 1971.

8. Thorsteinsson SB, Musher DM, Fagan T: The diagnostic value of sputum culture in acute pneumonia. JAMA 233:894-95, 1975.

9. Drew WL: Value of sputum culture in diagnosis of pneumococcal pneumonia. J Clin Microbiol 6:62-5, 1977.

10. Gerding DN: Etiologic diagnosis of acute pneumonia in adults - a growing challenge. Pneumonia Diag 69:136-50, 1981.

11. Baumgartner JD, Glausser MP: Single daily dose treatment of severe refractory infections with Ceftriaxone - cost savings and possible parenteral outpatient treatment. Arch Int Med 143:1868-73, 1983.

12. Tanner DJ: Cost containment for reconstituted parenteral antibiotics: Personnel and supply costs associated with their preparation, dispensing and administration. Review of Infectious Diseases (In Press).

\section{Acknowledgement}

The authors are appreciative of the efforts of the housestaff in fulfilling the various requirements of the protocol. The expert secretarial assistance of Ms. Terry Ishman is gratefully acknowledged. 